\title{
Unary Coding Controlled Simultaneous Wireless Information and Power Transfer
}

\author{
Jie Hu, Member, IEEE, Mengyuan Li, Kun Yang, Senior Member, IEEE, Soon Xin Ng, Senior Member, IEEE, \\ Kai-Kit Wong, Fellow, IEEE
}

\begin{abstract}
Radio frequency (RF) signals have been relied upon for both wireless information delivery and wireless charging to the massively deployed low-power Internet of Things (IoT) devices. Extensive efforts have been invested in physical layer and medium-access-control layer design for coordinating simultaneous wireless information and power transfer (SWIPT) in RF bands. Different from the existing works, we study the coding controlled SWIPT from the information theoretical perspective with practical transceiver. Due to its practical decoding implementation and its flexibility on the codeword structure, unary code is chosen for joint information and energy encoding. Wireless power transfer (WPT) performance in terms of energy harvested per binary sign and of battery overflow/underflow probability is maximised by optimising the codeword distribution of coded information source, while satisfying required wireless information transfer (WIT) performance in terms of mutual information. Furthermore, a Genetic Algorithm (GA) aided coding design is proposed to reduce the computational complexity. Numerical results characterise the SWIPT performance and validate the optimality of our proposed GA aided unary coding design.
\end{abstract}

Index Terms-SWIPT, unary coding, mutual information, energy per binary sign and battery overflow/underflow probability

\section{INTRODUCTION}

In the era of Internet of Things (IoT), massively deployed low-power devices may account for a major portion of wireless connectivity [1] for data collecting, data processing, data downloading and data uploading. However, frequent operations may quickly drain embedded batteries having limited capacity. Moreover, a lot of IoT devices are deployed in hardly reachable places. Replacing their batteries may substantially increase maintenance expenses. RF signal based wireless power transfer (WPT) is capable of delivering controllable,

Jie $\mathrm{Hu}$ and Mengyuan $\mathrm{Li}$ are with the School of Information and Communication Engineering, University of Electronic Science and Technology of China, Chengdu, China, 611731, email: hujie@uestc.edu.cn, 201721010518@std.uestc.edu.cn.

Kun Yang is both with the School of Information and Communication Engineering, University of Electronic Science and Technology of China, Chengdu, China, 611731, and with the School of Computer Science and Electronic Engineering, University of Esssex, Colchester, UK, CO4 3SQ, email: kunyang@uestc.edu.cn.

Soon Xin $\mathrm{Ng}$ is with the School of Electronics and Computer Science, University ofSouthampton, United Kingdom, SO17 1BJ, email: sxn@ecs.soton.ac.uk.

Kai-Kit Wong is with the Department of Electronic and Electrical Engineering, University College London, United Kingdom, WC1E 6BT, email: kai-kit.wong@ucl.ac.uk.

The authors would like to thank the financial support of National Natural Science Foundation of China (NSFC), Grant No. 61971102 and U1705263, that of GF Innovative Research Program as well as that of the Sichuan Science and Technology Program, No. 2019YJ0194. This work is also partially sponsored by Huawei Innovative Research Program (HIRP). reliable and flexible energy to remote low-power IoT devices [2]. However, wireless information transfer (WIT) has already resided in RF bands. Introducing WPT in the same band may severely degrade WIT performance, since they may compete for precious radio resources to fulfil their distinctive targets [3]. Therefore, carefully coordinating both WIT and WPT in RF bands yields an emerging technique of simultaneous wireless information and power transfer (SWIPT) [4].

A great deal of efforts have been invested in SWIPT spanning from signal and transceiver designs to mediumaccess-control strategies as well as resource allocation and networking schemes [5]-[14]. For example, Clerckx et al. [5] designed the optimal multi-sinusoidal signal for dedicated WPT, while Zeng et al. [6] focused on the design of multisinusoidal SWIPT signal. Zhou et al. [7] made the very first contribution to receiver arhictecture of SWIPT, which adopted either a power splitter, a time switcher or a current splitter for simultanous reception of information and energy. Moreover, Zhang et al. [8] optimised transmit beamformer for a MIMO aided SWIPT transmitter. Furthermore, Lv et al. [9] proposed an optimal time-domain resource allocation scheme for a timedivision-multiple-access (TDMA) aided multi-user SWIPT system, which maximised sum-throughput and fair-throughput of users' uplink transmissions, respectively. Based on [9], Yang et al. implemented multiple antennas at a SWIPT transmitter, which results in a joint resource allocation scheme [10] in the spatial-, time- and power-domain. Moreover, Gautam et al. [11] studied joint relay selection and sub-carrier allocation in an OFDMA aided multi-user SWIPT system. In order to support the "ad hoc" access of batteryless devices, Zhao et al. proposed several enhanced carrier-sensing-multiple-access and collision avoidance (CSMA/CA) protocols and analysed their attainable performance in [12] and [13]. Moreover, Fouladgar et al. [15] investigated the coordination of distributed wireless nodes for achieving the required quality of service (QoS) of SWIPT. Furthermore, Zhao et al. [14] studied an optimal deployment scheme of SWIPT transmitters by considering social characteristics of mobile SWIPT users. The impact of discrete messages on the SWIPT was discussed in [16], which firstly provided a design guide of the SWIPT coding and modulation.

Surprisingly, the research on the information theoretical essence of SWIPT has been comparatively slower, since Varshney [17] first studied the tradeoff between maximum mutual information and energy harvesting requirement in different channels. This seminal work demonstrated that SWIPT performance can be controlled by adjusting the codeword structure, 
which provided the theoretical fundamental for coding controlled SWIPT. Furthermore, Varshney [18] also proposed a cross-layer architecture for jointly optimising both the energy and information delivery from the information theoretical perspective, where a powerline communication system was exemplified as a typical integrated information and energy transfer system. Popovski et al. [19] derived both the inner and outer bounds of information transmission rate in a two-way noiseless channel, where the energy contained in the received signal was reused for powering the successive communications. Then, Yin et al. [20] studied a variable-length coding design for SWIPT in a binary noiseless channel. Moreover, Tandon et al. [21] divided a codeword into several sub-blocks for satisfying the receiver's real-time energy demands. The optimal structure of codewords was found for maximising the mutual information in a binary symmetric channel (BSC). However, both of [17] and [21] only considered memoryless information source in their analysis but ignored the correlation among the binary signs in the output sequence of a practical encoder. Therefore, these works optimised transmit probabilities of binary signs in order to achieve the maximum SWIPT performance without considering a practical coding scheme.

Conventional source encoders generate equi-probable binary signs for maximising codewords' capability of information transmission. However, codewords having equi-probable binary signs are only capable of carrying a fixed amount of energy. Therefore, conventional source encoders cannot satisfy diverse energy requests of receivers. Constrained code [22] has a certain degree of freedom to adjust codeword structure for satisfying specific WPT requirements without sacrificing WIT efficiency.

As a typical constrained code, a run-length-limited (RLL) encoder was designed by Fouladgar et al. [23] for minimising battery overflow/underflow probability at the receiver subject to a constraint on the achievable mutual information. Moreover, Tandon et al. [24] found the capacity lower-bound of BSC and Z-channel, when a $(d, \infty)$ RLL code was adopted. However, they only used the minimum run-length $d$ to ensure the minimum energy delivery requirement, which largely overlooks devices' long-term energy requirements.

Furthermore, unary code has been widely used for source coding [25]-[27] in wireless communication, due to its low encoding and decoding complexity. Specifically, unary code was used by Babar et al. [25] for joint throughput and dimming control in visible light communication system. However, the SWIPT performance of unary code in the RF band is not well understood from the information theoretical perspective.

Against this background, our novel contributions are summarised as below:

- We propose a practical unary coding aided SWIPT transceiver and analysed its performance from the information theoretical perspective. The correlation among the information signs generated by the unary encoder has been taken into account in the performance analysis.

- By considering infinite battery capacity, the codeword distribution is optimised for maximising the average energy harvested from a single sign received by an energy
TABLE I

Notation List

\begin{tabular}{ll}
\hline Notation & Definition \\
\hline $\mathbb{X} / \widehat{\mathbb{X}}$ & Original/coded information source \\
$\mathbb{Y} / \widehat{\mathbb{Y}}$ & Original/coded information destination \\
$\mathcal{X}_{m} / \widehat{X}_{k}$ & $m$-th message/ $k$-th codeword \\
$p\left(\mathcal{X}_{m}\right) / p\left(\widehat{X}_{k}\right)$ & Probability of $m$-th message/ $k$-th codeword \\
$\boldsymbol{X} / \widehat{\mathbb{X}}$ & Message/code book \\
$\mathbf{P}(\widehat{\mathbb{X}}) / \mathbf{P}(\widehat{\mathbb{X}})$ & Message/Codeword Distribution \\
$\omega_{I} / \omega_{E}$ & Crossover prob. of WIT/WPT channel \\
$K$ & Level of Unary code \\
$\mathbf{S}_{N}$ & State sequence of Markov chain \\
$\mathbf{X}_{N} / \mathbf{Y}_{N}$ & Channel input/output binary sign sequence \\
$q_{n}$ & Transition prob. from state $n$ to $(n+1)$ \\
$\mathbf{Q}$ & Matrix of state transition probabilities \\
$\pi_{n}$ & Steady-state probability of state $n$ \\
$\mathcal{\mathbb { E }}$ & Energy destination \\
$I_{\text {inf }}(\widehat{\mathbb{X}} ; \widehat{\mathbb{Y}})$ & Energy harvested per received binary sign \\
$B(t)$ & Inf of mutual information between $\widehat{\mathbb{X}}$ and $\widehat{\mathbb{Y}}$ \\
$\lambda(t) / \mu(t)$ & Energy level in battery at the $t$-th instant \\
$\xi_{B, B+b}$ & Energy arrival/departure at the $t$-th instant \\
$\widehat{\pi}$ & Trans. prob. from energy level $B$ to $B+b$ \\
$B_{\max } / B_{\text {min }}$ & Steady-state probability of energy level $B$ \\
$p_{o f} / p_{u f}$ & Upper/lower bound of battery energy level \\
\hline & Battery overflow/underflow probability \\
\hline &
\end{tabular}

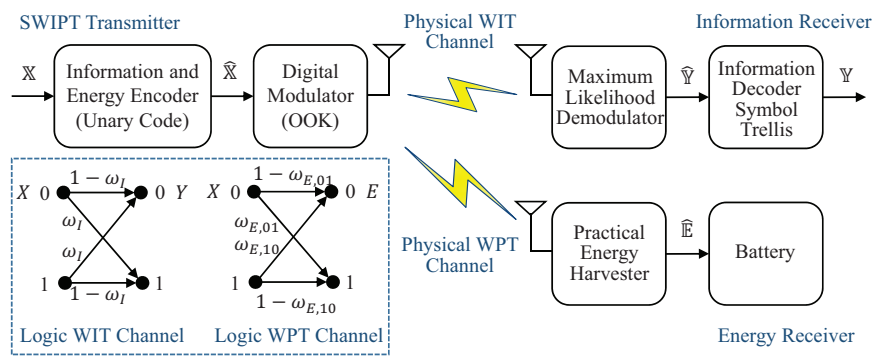

Fig. 1. Practical transceiver for coding controlled SWIPT.

receiver (ER), while satisfying the mutual information requirement of an information receiver (IR).

- By also considering finite battery capacity, the optimal codeword distribution is found for minimising the battery overflow/underflow probability of an ER, while satisfying the mutual information requirement of an IR.

- The optimal coding design is obtained by exploiting a low-complexity Genetic Algorithm (GA), which achieves almost the same performance of the exhaustive search (ES).

The rest of the paper is organised as follows: a practical unary coding aided SWIPT transceiver is introduced in Section II. The WIT and WPT performance is analysed in Section III and IV, respectively. After formulating and solving the optimal coding design problem in Section $\mathrm{V}$, numerical results are illustrated in Section VI. Finally, our paper is concluded in Section VII. All the notations are summarised in TABLE I.

\section{System Model}

\section{A. SWIPT Transmitter}

As portrayed in Fig. 1, a SWIPT transmitter is constituted by the following functional modules: 
TABLE II

$K$-Level Unary Code

\begin{tabular}{llll}
\hline Messages & Message Prob. & Codewords & Codeword Prob. \\
\hline$X_{1}$ & $p\left(\mathcal{X}_{1}\right)$ & $\widehat{X}_{1}=0$ & $p\left(\widehat{X}_{1}\right)$ \\
$X_{2}$ & $p\left(X_{2}\right)$ & $\widehat{X}_{2}=10$ & $p\left(\widehat{X}_{2}\right)$ \\
$X_{3}$ & $p\left(X_{3}\right)$ & $\widehat{X}_{3}=110$ & $p\left(\widehat{X}_{3}\right)$ \\
$\vdots$ & $\vdots$ & $\vdots$ & $\vdots$ \\
$X_{M}$ & $p\left(X_{M}\right)$ & $\widehat{X}_{K}=\underbrace{11 \cdots 1}_{(K-1) \text { sign }} 0$ & $p\left(\widehat{X}_{K}\right)$ \\
\hline
\end{tabular}

- Information and Energy Encoder adopts the $K$-level unary coding for mapping $M$ messages $\mathcal{X}=\left\{\mathcal{X}_{1}, \cdots, \mathcal{X}_{M}\right\}$ randomly generated by the information source $\mathbb{X}$ onto $K$ codewords $\left\{\widehat{X}_{1}, \cdots, \widehat{X}_{K}\right\}$, where the $k$-th codeword $\widehat{X}_{k}$ has $(k-1)$ signs ' 1 ' followed by a sign ' 0 ', as portrayed in TABLE II. The resultant coded information source is denoted as $\widehat{\mathbb{X}}$. Given a specific message distribution $\mathbf{P}(\mathbb{X})=\left[p\left(\mathcal{X}_{1}\right), \cdots, p\left(\mathcal{X}_{M}\right)\right]$ of $\mathbb{X}$, we may obtain an arbitrary codeword distribution $\mathbf{P}(\widehat{\mathbb{X}})=\left[p\left(\widehat{X}_{1}\right), \cdots, p\left(\widehat{X}_{K}\right)\right]$ by exploiting the equation $\mathbf{P}(\widehat{\mathbb{X}})=\mathbf{P}(\widehat{\mathbb{X}}) \times \mathbf{P}(\widehat{\mathbb{X}} \mid \mathbb{X})$, where the matrix $\mathbf{P}(\widehat{\mathbb{X}} \mid \mathbb{X})=\left[p\left(\widehat{\mathcal{X}}_{k} \mid \mathcal{X}_{m}\right)\right]_{M \times K}$ is carefully chosen. The entry $p\left(\widehat{X}_{k} \mid X_{m}\right)$ is the probability of mapping the message $\mathcal{X}_{m}$ onto the codeword $\widehat{X}_{k}^{1}$.

- Digital Modulator modulates the binary codewords onto the analogue RF signals. Amplitude based modulator allocates different power to the codewords. In order to highlight the impact of the codeword structure on the SWIPT performance, we adopt an on-off-keying (OOK) based digital modulator. Binary sign ' 1 ' is represented by the presence of the RF signal $\sqrt{P_{t}} \sin \left(2 \pi f_{c} t\right)$, where $P_{t}$ and $f_{c}$ denote the transmit power and the carrier frequency, respectively. By contrast, sign ' 0 ' is represented by the absence of the RF signal.

Discussion: traditional information encoder aims for maximising the mutual information, which ignores the QoS of WPT performance. By contrast, information and energy encoder achieves a balance between the WIT and WPT performance. The resultant optimal codeword distribution may sacrifice some WIT performance in order to satisfy the QoS of WPT.

\section{B. Information and Energy Receiver}

An information receiver (IR) consists of the following modules, as illustrated in Fig. 1:

- Digital Demodulator demodulates the information carried by the received RF signal by adopting the maximumlikelihood (ML) principle. The resultant binary sign sequences constitute the coded information destination $\widehat{\mathbb{Y}}$.

- Information Decoder recovers the original message from the received codeword by exploiting the symbol-level trellis [25]-[27], which yields the information destination Y.

\footnotetext{
${ }^{1}$ By carefully adjusting the entries in the encoding matrix $\mathbf{P}(\widehat{\mathbb{X}} \mid \mathbb{X})$, we may achieve either a lossless or a lossy source coding scheme.
}

Due to the broadcast nature of wireless channels, the RF signal transmitted by the SWIPT transmitter can also be received by an energy receiver (ER) for energy harvesting, which consists of the following modules, as illustrated in Fig. 1:

- Energy Harvester converts the RF signal received to the direct current (DC). A single energy unit can be harvested, when the input RF power is higher than $P_{t h}$. Binary notation $E=1$ represents that the ER harvests a single energy unit, while $E=0$ represents no energy harvested, which constitutes an energy destination $\widehat{\mathbb{E}}$.

- Battery is charged by the DC. We consider both infiniteand finite-capacity based batteries in the optimal coding design.

Discussion: the IR and ER are either co-located or separated for simultaneous information reception and energy harvesting.

\section{WIT and WPT Channel}

1) WIT Channel Modelling: A logic WIT channel connecting the SWIPT transmitter and the IR consists of an OOK based modulator, a physical wireless channel and an ML based demodulator. The RF signal received by the IR is either $r_{I}=A_{I}+z_{I}$, if the binary $\operatorname{sign} X=1$ is input to the channel, or $r_{I}=z_{I}$, if the channel input is $X=0$. Specifically, $A_{I}=\sqrt{P_{t} / \Omega\left(d_{I}\right)}$ is the amplitude of the modulated RF signal received by the IR, where $\Omega\left(d_{I}\right)$ is the path-loss since the IR is $d_{I} \mathrm{~m}$ away from the SWIPT transmitter, while $z_{I} \sim \mathcal{N}\left(0, \sigma_{I}^{2}\right)$ is the random ambient RF interference at the IR.

By adopting the ML based demodulator, the crossover probability from the channel input $X=0$ to the output $Y=1$ and that from $X=1$ to $Y=0$ are both equal to $\omega_{I}=Q\left(\frac{A_{I}}{2 \sigma_{I}}\right)$, where $Q(\cdot)$ is the tail distribution function of a standard Gaussian distribution. Therefore, the logic WIT channel can be modelled as a binary symmetric channel (BSC), as portrayed in Fig. 1.

2) WPT Channel Modelling: A logic WPT channel connecting the SWIPT transmitter and the ER consists of an OOK based modulator, a physical wireless channel and a practical energy harvester. The RF signal received by the ER is either $r_{E}=A_{E}+z_{E}$, if the binary $\operatorname{sign} X=1$ is input to the channel, or $r_{E}=z_{E}$, if the channel input is $X=0$. Specifically, $A_{E}=\sqrt{P_{t} / \Omega\left(d_{E}\right)}$ is the amplitude of the modulated RF signal received by the IR, where $\Omega\left(d_{E}\right)$ is the path-loss since the ER is $d_{E} \mathrm{~m}$ away from the SWIPT transmitter, while $z_{E} \sim \mathcal{N}\left(0, \sigma_{E}^{2}\right)$ is the random RF interference at the ER.

By considering a practical energy harvester, the crossover probability $\omega_{E, 01}$ from the channel input $X=0$ to the channel output $E=1$ and that $\omega_{E, 10}$ from $X=1$ to $E=0$ are expressed as

$$
\left\{\begin{array}{l}
\omega_{E, 01}=2 Q\left(\frac{\sqrt{P_{t h}}}{\sigma_{E}}\right), \\
\omega_{E, 10}=1-Q\left(\frac{\sqrt{P_{t h}}+A_{E}}{\sigma_{E}}\right)-Q\left(\frac{\sqrt{P_{t h}}-A_{E}}{\sigma_{E}}\right),
\end{array}\right.
$$

respectively. Therefore, the logic WPT channel can be modelled as a binary asymmetric channel (BAC), as portrayed in Fig. 1.

Discussion: Different from physical SWIPT channels adopted in the physical layer design [5]-[14], we conceive 


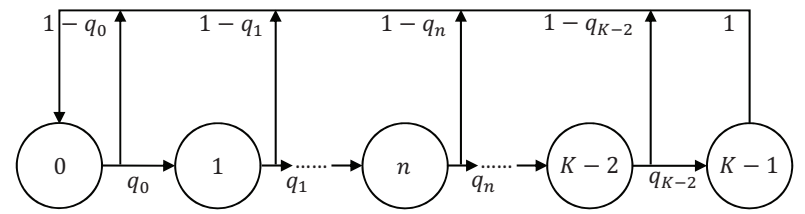

Fig. 2. Markov modelling of the unary coded information source $\widehat{\mathbb{X}}$.

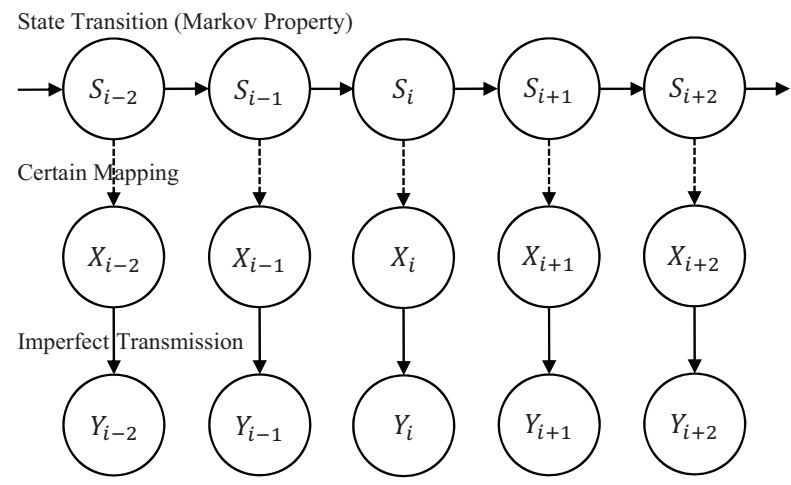

Fig. 3. Correlation among the state sequence and the sign sequences of the channel input/output.

logic WIT and WPT channels by jointly considering the impact of modulation on WIT and the impact of energy harvester's sensitivity on WPT.

\section{WIT Performance Analysis}

\section{A. Markov Modelling}

The $K$-level unary encoded information source $\widehat{\mathbb{X}}$ sequentially sends a range of codewords chosen from the codebook $\widehat{\mathcal{X}}$ for simultaneously conveying information to the IR and transferring energy to the ER, which generates an $N$-sign sequence $\mathbf{X}_{N}=\left\{X_{1}, X_{2}, \cdots, X_{N}\right\}$. The correlation among the binary signs is modelled by a finite-state Markov chain, as portrayed in Fig. 2.

State $n$ of this Markov chain represents that the unary coded information source $\widehat{\mathbb{X}}$ has already output a run of sign ' 1 ' having a length of $m$ at the current instant, since $\widehat{\mathbb{X}}$ is in the process of sending an arbitrary codeword chosen from $\left\{\widehat{X}_{n+1}, \widehat{X}_{n+2}, \cdots, \widehat{X}_{K}\right\}$. By considering the codeword structure of TABLE II, if $\widehat{\mathbb{X}}$ is in the process of sending codewords $\left\{\widehat{X}_{n+2}, \cdots, \widehat{X}_{K}\right\}$, the next output sign is ' 1 '. The run-length of sign ' 1 ' is then updated to $(n+1)$, which results in the state transition from $n$ to $(n+1)$. The corresponding state transition probability is expressed as

$$
q_{n}=\frac{p\left(\widehat{X}_{n+2}\right)+\cdots+p\left(\widehat{X}_{K}\right)}{p\left(\widehat{X}_{n+1}\right)+p\left(\widehat{X}_{n+2}\right)+\cdots+p\left(\widehat{X}_{K}\right)},
$$

for $0 \leq n \leq K-2$. The probability of the Markov chain transiting from state $n$ back to state 0 is thus $\left(1-q_{n}\right)$. Specifically, when the Markov chain stays at state $(K-1)$, it transits back to state 0 at the next instant for certain, since $(K-1)$ is the maximum allowable run-length of sign ' 1 ' for a $K$-level unary code, as shown in TABLE II.
The matrix $\mathbf{Q}$ containing all the state transition probabilities is expressed as:

$$
\mathbf{Q}=\left[\begin{array}{llllll}
1-q_{0} & q_{0} & 0 & \cdots & 0 & 0 \\
1-q_{1} & 0 & q_{1} & \cdots & 0 & 0 \\
\vdots & \vdots & \vdots & \ddots & \vdots & \vdots \\
1-q_{K-2} & 0 & 0 & \cdots & 0 & q_{K-2} \\
1 & 0 & 0 & \cdots & 0 & 0
\end{array}\right]
$$

The stationary distribution $\pi=\left\{\pi_{0}, \pi_{1}, \cdots, \pi_{K-1}\right\}$ of the Markov chain can be obtained by solving the following linear equations:

$$
\left\{\begin{array}{l}
\boldsymbol{\pi} \times \mathbf{Q}=\boldsymbol{\pi} \\
\boldsymbol{\pi} \times \mathbf{I}_{K \times 1}=1
\end{array},\right.
$$

where $\mathbf{I}_{K \times 1}$ is a $K \times 1$ column vector having all its entries equal to one.

A state sequence $\mathbf{S}_{N}=\left\{S_{1}, \cdots, S_{N}\right\}$ can be mapped onto a sign sequence $\mathbf{X}_{N}=\left\{X_{1}, \cdots, X_{N}\right\}$, as portrayed in Fig. 3 . For an example of a 4-level unary code, a 6-states sequence $\mathbf{S}_{6}=\{1,2,0,1,2,3,0\}$ corresponds to a sign sequence $\mathbf{X}_{6}=$ $\{1,1,0,1,1,1,0\}$.

\section{B. Mutual Information}

When the $N$-sign sequence $\mathbf{X}_{N}$ is input to the channel, the corresponding output sign sequence is $\mathbf{Y}_{N}=\left\{Y_{1}, Y_{2}, \cdots, Y_{N}\right\}$ after the imperfect transmission, as exemplified in Fig.3. The mutual information between the coded source $\widehat{\mathbb{X}}$ and the coded destination $\widehat{\mathbb{Y}}$ can be formulated as

$$
I(\widehat{\mathbb{X}} ; \widehat{\mathbb{Y}})=H(\widehat{\mathbb{X}})-H(\widehat{\mathbb{X}} \mid \widehat{\mathbb{Y}}) .
$$

The entropy $H(\widehat{\mathbb{X}})$ of the coded source can be expressed as

$$
\begin{aligned}
H(\widehat{\mathbb{X}}) & =\lim _{N \rightarrow \infty} \frac{H\left(\mathbf{X}_{N}\right)}{N} \stackrel{(a)}{=} \lim _{N \rightarrow \infty} \frac{H\left(\mathbf{S}_{N}\right)}{N} \\
& \stackrel{(b)}{=} \lim _{N \rightarrow \infty} H\left(S_{N} \mid S_{1}, S_{2}, \cdots, S_{N-1}\right) \\
& \stackrel{(c)}{=} \lim _{N \rightarrow \infty} H\left(S_{N} \mid S_{N-1}\right) \stackrel{(d)}{=} H\left(S_{2} \mid S_{1}\right) .
\end{aligned}
$$

In Eq. (6), the equality $(a)$ is derived due to the certain mapping between the sign sequence $\mathbf{X}_{N}$ and the state sequence $\mathbf{S}_{N}$, as exemplified in Fig. 3. The equality $(b)$ is obtained by exploiting Theorem 4.2.1 of [28]. The equality $(c)$ is derived due to the Markov property of $\overrightarrow{\mathbb{X}}$, while the derivation of the equality $(d)$ is based on the stationary property of $\widehat{\mathbb{X}}$.

In order to obtain the conditional entropy $H(\widehat{\mathbb{X}} \mid \widehat{Y})$, the following pair of Lemmas are introduced for investigating the correlation between $\mathbf{S}_{N}$ and $\mathbf{Y}_{N}$ :

Lemma 1: Given state $S_{i}$ at the $i$-th instant, state $S_{i+1}$ is independent of the channel output signs $\left\{Y_{1}, \cdots, Y_{i-1}, Y_{i}\right\}$ at the previous instants.

Proof: Please refer to Appendix A.

Lemma 2: State $S_{i}$ at the $i$-th instant is correlated to the channel output signs $\left\{Y_{i+1}, Y_{i+2}, \ldots\right\}$ at the subsequent instants. If the state transition probabilities satisfy $\left\{q_{n}=\right.$ $q \mid n=0,1, \cdots, K-2\}$, where $q \in(0,1)$ is a constant, $S_{i}$ is independent of $\left\{Y_{i+1}, Y_{i+2}, \cdots\right\}$.

Proof: Please refer to Appendix B. 
Therefore, the conditional entropy $H(\widehat{\mathbb{X}} \mid \widehat{\mathbb{Y}})$ is calculated as

$$
\begin{aligned}
& H(\widehat{\mathbb{X}} \mid \widehat{\mathbb{Y}})=\lim _{N \rightarrow \infty} \frac{H\left(\mathbf{X}_{N} \mid \mathbf{Y}_{N}\right)}{N}=\lim _{N \rightarrow \infty} \frac{H\left(\mathbf{S}_{N} \mid \mathbf{Y}_{N}\right)}{N} \\
& \stackrel{(e)}{=} \lim _{N \rightarrow \infty} \frac{1}{N}\left[H\left(S_{1} \mid Y_{1}, \cdots, Y_{N}\right)\right. \\
& \left.\quad+\sum_{i=2}^{N} H\left(S_{i} \mid S_{i-1}, \cdots, S_{1}, Y_{1}, \cdots, Y_{N}\right)\right] \\
& \stackrel{(f)}{=} \lim _{N \rightarrow \infty} \frac{1}{N}\left[H\left(S_{1} \mid Y_{1}, \cdots, Y_{N}\right)+\sum_{i=2}^{N} H\left(S_{i} \mid S_{i-1}, Y_{i}, \cdots, Y_{N}\right)\right] \\
& \quad \stackrel{(g)}{\leq} \lim _{N \rightarrow \infty} \frac{H\left(S_{1} \mid Y_{1}, \cdots, Y_{N}\right)+(N-1) H\left(S_{2} \mid S_{1}, Y_{2}\right)}{N} \\
& =H\left(S_{2} \mid S_{1}, Y_{2}\right) .
\end{aligned}
$$

By exploiting the chain rule for entropy (Theorem 2.5.1 in [28]), we obtain the equality ( $e$ ) of Eq. (7). The equality $(f)$ is derived by exploiting i) the Markov property and ii) Lemma 1. According to Lemma 2, State $S_{i}$ is dependent of the channel output signs $\left\{Y_{i}, Y_{i+1}, \cdots, Y_{N}\right\}$, which results in the inequality $(g)$.

By substituting Eqs.(6) and (7) into (5), the mutual information is finally derived as

$$
\begin{aligned}
I(\widehat{\mathbb{X}} ; \widehat{\mathbb{Y}}) & \stackrel{(h)}{\geq} H\left(S_{2} \mid S_{1}\right)-H\left(S_{2} \mid S_{1}, Y_{2}\right) \\
= & I\left(S_{2} ; Y_{2} \mid S_{1}\right)=H\left(Y_{2} \mid S_{1}\right)-H\left(Y_{2} \mid S_{2}, S_{1}\right) \\
& =-\sum_{s_{1}} \sum_{y_{2}} p\left(y_{2}, s_{1}\right) \log _{2} p\left(y_{2} \mid s_{1}\right) \\
& \quad+\sum_{s_{1}} \sum_{s_{2}} \sum_{y_{2}} p\left(y_{2}, s_{2}, s_{1}\right) \log _{2} p\left(y_{2} \mid s_{2} s_{1}\right) \\
& \stackrel{(i)}{=} \sum_{n=0}^{K-1} \pi_{n}\left\{H\left[q_{n} \omega_{I}+\left(1-q_{n}\right)\left(1-\omega_{I}\right)\right]-H\left(\omega_{I}\right)\right\} \\
& \triangleq
\end{aligned}
$$

where $H(x)=-x \log _{2}(x)-(1-x) \log _{2}(1-x)$ for $0<x<1$. The inequality $(h)$ of Eq. (8) is obtained by substituting the upper bound $H\left(S_{2} \mid S_{1}, Y_{2}\right)$ of the conditional entropy $H(\widehat{\mathbb{X}} \mid \widehat{\mathbb{Y}})$. We further derive the equality $(i)$ by exploiting the stationary distribution $\boldsymbol{\pi}$ of the Markov chain and the corresponding matrix $\mathbf{Q}$ of the transition probabilities. The lower bound of $I(\widehat{\mathbb{X}} ; \widehat{Y})$ derived in Eq. (8) is attainable, according to Lemma 2. Therefore, it is the infimum of $I(\widehat{\mathbb{X}} ; \widehat{\mathbb{Y}})$ denoted as $I_{\text {inf }}(\widehat{\mathbb{X}} ; \widehat{\mathbb{Y}})$, which is a function of the codeword distribution $\mathbf{P}(\widehat{\mathbb{X}})$, since entries in both $\mathbf{Q}$ and $\pi$ are functions of $\mathbf{P}(\widehat{\mathbb{X}})$, according to Eqs. (2) and (4).

\section{WPT Performance Analysis}

\section{A. Infinite Battery Capacity}

When the battery has infinite capacity, we focus on the average energy harvested from a single sign received by the ER.

When the Markov chain of Fig. 2 stays at state $n$, the $K$-level unary coded source $\widehat{\mathbb{X}}$ may output a sign ' 1 ' with a probability of $q_{n}$, while it may output a sign ' 0 ' with a probability of $\left(1-q_{n}\right)$. Given the stationary distribution $\pi$ derived by solving Eq. (4), the stationary probability of

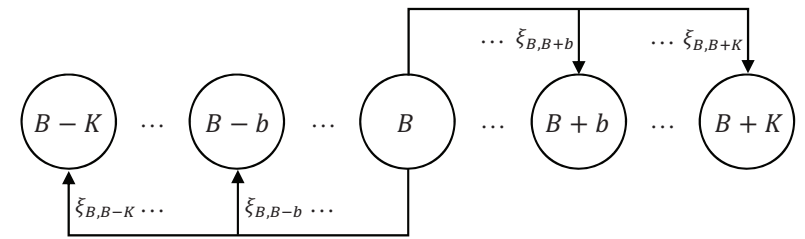

Fig. 4. Markov modelling of the battery queuing process $B(t)$.

$\widehat{\mathbb{X}}$ outputting a sign ' 1 ' is expressed as $p_{1}=\sum_{n=0}^{K-1} \pi_{n} q_{n}$. Similarly, the stationary probability of $\widehat{\mathbb{X}}$ outputting a sign ' 0 ' is expressed as $p_{0}=\sum_{n=0}^{K-1} \pi_{n}\left(1-q_{n}\right)$. Therefore, after the imperfect transmission in the WPT channel of Fig. 1, the average energy harvested from a single sign received by the $\mathrm{ER}$ is formulated as

$$
\begin{aligned}
\mathcal{E}(\widehat{\mathbb{E}}) & =p_{1}\left(1-\omega_{E, 10}\right)+p_{0} \omega_{E, 01} \\
& =\sum_{n=0}^{K-1} \pi_{n}\left[q_{n}\left(1-\omega_{E, 10}\right)+\left(1-q_{n}\right) \omega_{E, 01}\right],
\end{aligned}
$$

which is a function of the codeword distribution $\mathbf{P}(\widehat{\mathbb{X}})$, since entries in both $\mathbf{Q}$ and $\boldsymbol{\pi}$ are all functions of $\mathbf{P}(\widehat{\mathbb{X}})$, according to Eqs. (2) and (4).

\section{B. Finite Battery Capacity}

When the battery has a finite capacity $B_{\max }$ and a warning threshold $B_{\min }$, we study both the battery overflow probability $p_{o f}$ and the battery underflow probability $p_{u f}$ of the ER.

The instantaneous energy level can be modelled by the following queuing process:

$$
B(t+1)=\left\{\begin{array}{l}
\max \left\{B_{\min }, B(t)+\lambda(t)-\mu(t)\right\}, \\
\min \left\{B(t)+\lambda(t)-\mu(t), B_{\max }\right\},
\end{array}\right.
$$

where $B(t)$ is the energy level of the battery at the $t$-th instant, $\lambda(t)$ is the amount of energy arriving at the battery of the ER at the $t$-th instant and $\mu(t)$ is the amount of energy departing from the battery at the $t$-th instant. The energy level $B(t+1)$ at the next instant is lower-bounded by $B_{\min }$ and upper-bounded by $B_{\max }$. At a single instant, an arbitrary unary codeword is sent by the SWIPT transmitter.

Specifically, the energy arrival $\lambda(t)$ is a stochastic process, which has the following randomness:

- A codeword $\widehat{X}_{k}$ is randomly sent by the SWIPT transmitter with a probability of $p\left(\widehat{X}_{k}\right)$, which includes $(k-1)$ signs ' 1 ' carrying energy.

- The binary signs in $\widehat{X}_{k}$ may be randomly flipped by the logic WPT channel of Fig. 1.

We assume that the probability of the ER consuming a single energy unit in a sign duration is $v$. Therefore, if a codeword $\widehat{X_{k}}$ is sent by the SWIPT transmitter at the $t$-th instant, the amount of energy $\mu(t)$ consumed by the ER obeys a Binomial distribution having parameters of $(k, v)$.

The queuing process $B(t)$ can be further modelled by another discrete Markov chain, as illustrated in Fig. 4. The states in this Markov chain span from $B_{\min }$ to $B_{\max }$, which represent all possible energy levels of the battery. We now focus on deriving state transition probabilities. The probability 
$\xi_{b}$ of the energy level increment $b=\lambda(t)-\mu(t)$ is expressed as

$$
\begin{aligned}
\xi_{b} & =\sum_{\widehat{X}_{k}} \sum_{\lambda} p\left(\mu(t)=\lambda(t)-b, \lambda(t)=\lambda, \widehat{X}_{k}\right) \\
& =\sum_{k=1}^{K} p\left(\widehat{X}_{k}\right) \sum_{\lambda} p\left(\mu(t)=\lambda-b \mid \lambda(t)=\lambda, \widehat{X}_{k}\right) p\left(\lambda(t)=\lambda \mid \widehat{X}_{k}\right) .
\end{aligned}
$$

Given the unary codeword $\widehat{X}_{k}$ sent at the $t$-th instant, the conditional probability of $\lambda(t)=\lambda$ is expressed as

$$
\begin{aligned}
p\left(\lambda(t)=\lambda \mid \widehat{X}_{k}\right) & =\left(\begin{array}{l}
k \\
\lambda
\end{array}\right) \frac{\left(1-\omega_{E, 10}\right)^{\lambda-1} \omega_{E, 10}^{k-\lambda}}{k} \\
\cdot & {\left[(k-\lambda)\left(1-\omega_{E, 10}\right)\left(1-\omega_{E, 01}\right)+\lambda \omega_{E, 10} \omega_{E, 01}\right], }
\end{aligned}
$$

for $0 \leq \lambda \leq k$, and $p\left(\lambda(t)=\lambda \mid \widehat{X}_{k}\right)=0$, otherwise. Given the unary codeword $\widehat{X}_{k}$ and $\lambda(t)=\lambda$, the conditional probability $p\left(\mu(t)=\lambda-b \mid \widehat{X}_{k}, \lambda(t)=\lambda\right)$ is calculated as

$$
\begin{aligned}
p(\mu(t) & \left.=\lambda-b \mid \widehat{\mathcal{X}}_{k}, \lambda(t)=\lambda\right) \\
& = \begin{cases}\left(\begin{array}{c}
k \\
\lambda-b
\end{array}\right) v^{\lambda-b}(1-v)^{k-\lambda+b}, & 0 \leq \lambda-b \leq k, \\
0, & \text { otherwise. }\end{cases}
\end{aligned}
$$

By substituting both Eqs. (12) and (13) into (11), we may obtain $\xi_{b}$ as

$$
\xi_{b}=\sum_{k=1}^{K} p\left(\widehat{X}_{k}\right) \Phi(b, k), \text { for }-K \leq b \leq K,
$$

and $\xi_{b}=0$, otherwise, where we have $\Phi(b, k)$ expressed in Eq. (15) on the next page.

Without loss of generality, we assume that the battery has an energy level of $B(t)=B$ at the $t$-th instant. Therefore, at the $(t+1)$-th instant, the energy level of the battery could be $B(t+1)=B+b$, which also represents the state transition from $B$ to $(B+b)$ in the Markov chain of Fig.4. The corresponding state transition probability $\xi_{B, B+b}$ can be then expressed as

$\xi_{B, B+b}=\left\{\begin{array}{cl}\xi_{b}, & \max \left(B_{\min }-B+1,-K\right) \leq b \leq \min \left(B_{\max }-B-1, K\right) \\ & \text { and } B_{\min }+1 \leq B \leq B_{\max }-1, \\ 0, & K<b \leq B_{\max }-B \text { and } B_{\min } \leq B<B_{\max }-K, \\ 0, & B_{\min }-B \leq b<-K \text { and } B_{\min }+K<B \leq B_{\max } .\end{array}\right.$

If the current energy level $B$ satisfies $B_{\max }-K \leq B \leq B_{\max }$ and the energy level increment $b$ satisfies $B_{\max }-B \leq b \leq K$, the energy level of the battery at the end of this instant is constrained to $B_{\max }$, according to Eq. (10). Therefore, the state transition probability $\xi_{B, B_{\max }}$ is expressed as

$$
\xi_{B, B_{\max }}=\sum_{b=B_{\max }-B}^{K} \xi_{b}, \text { for } B_{\max }-K \leq B \leq B_{\max } .
$$

Similarly, if the current energy level satisfies $B_{\min } \leq B \leq B_{\min }+$ $K$ and the energy level increment $b$ satisfies $-K \leq b \leq B_{\min }-B$, the energy level of the battery at the end of this instant is constrained to $B_{\min }$, according to Eq. (10). Therefore, the state transition probability $\xi_{B, B_{\min }}$ is expressed as

$$
\xi_{B, B_{\min }}=\sum_{b=-K}^{B_{\min }-B} \xi_{b}, \text { for } B_{\min } \leq B \leq B_{\min }+K .
$$

With the aid of Eqs. (16)-(18), the state transition probability matrix $\Xi=\left[\xi_{i, j}\right]$ is found. By following the similar method of Eq. (4), the stationary distribution $\widehat{\pi}=$ $\left\{\widehat{\pi}_{B_{\min }}, \cdots, \widehat{\pi}_{B}, \cdots, \widehat{\pi}_{B_{\max }}\right\}$ is obtained for all the states in the Markov chain of Fig.4. Finally, the battery overflow probability $p_{o f}$ and the underflow probability $p_{u f}$ are then formulated as

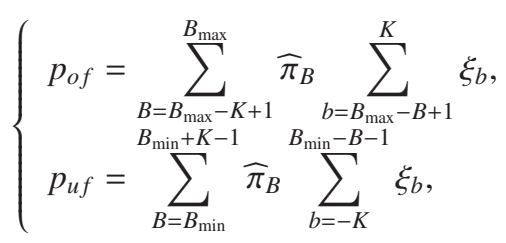

According to Eq. (14), both $p_{o f}$ and $p_{u f}$ are the functions of the codeword distribution $\mathbf{P}(\widehat{\mathbb{X}})$.

\section{Optimal Coding Design}

\section{A. Problem Formulation}

We formulate the first optimal coding design by considering an infinite battery capacity at the ER, when the $K$-level unary code is adopted at the SWIPT transmitter:

$$
\begin{aligned}
& \text { (P1): } \max _{\mathbf{P}(\widehat{\mathbb{X}})} \mathcal{E}(\widehat{\mathbb{E}}), \\
& \text { s. t.: } I_{\text {inf }}(\widehat{\mathbb{X}} ; \widehat{\mathbb{Y}}) \geq R_{t h} .
\end{aligned}
$$

The objective of (P1) is to maximise the average energy $\mathcal{E}(\widehat{\mathbb{E}})$ of Eq. (9) harvested from a single received sign by finding the optimal codeword distribution $\mathbf{P}_{1}^{*}(\widehat{\mathbb{X}})$, while satisfying the constraint (20a) that the mutual information's infinimum $I_{\text {inf }}(\widehat{\mathbb{X}} ; \widehat{\mathbb{Y}})$ of Eq. (8) should be higher than a rate threshold $R_{t h}$. Since we have $R=I(\widehat{\mathbb{X}} ; \widehat{\mathbb{Y}}) \geq I_{\text {inf }}(\widehat{\mathbb{X}} ; \widehat{\mathbb{Y}})$, the actual information transmission rate $R$ is also higher than $R_{t h}$. We use $\mathbf{P}_{1}^{*}$ to represent $\mathbf{P}_{1}^{*}(\widehat{\mathbb{X}})$ in Section V-B.

We then formulate the second optimal coding design by considering a finite battery capacity at the ER:

$$
\begin{aligned}
& \text { (P2): } \min _{\mathbf{P}(\widehat{\mathbb{X}})} p_{o f}\left(\text { or } p_{u f}\right), \\
& \text { s. t.: } I_{\text {inf }}(\widehat{\mathbb{X}} ; \widehat{\mathbb{Y}}) \geq R_{t h} .
\end{aligned}
$$

In contrast to $(\mathrm{P} 1)$, we aim for minimising the battery overflow/underflow probability in (P2) by finding the optimal codeword distribution $\mathbf{P}_{2}^{*}(\widehat{\mathbb{X}})$, while satisfying the minimum rate requirement $R_{t h}$. We use $\mathbf{P}_{2}^{*}$ to represent $\mathbf{P}_{2}^{*}(\widehat{\mathbb{X}})$ in Section V-C.

\section{B. ES Aided Coding Design}

The calculations of $I_{\text {inf }}(\widehat{\mathbb{X}} ; \widehat{\mathbb{Y}})$ and $\mathcal{E}(\widehat{\mathbb{E}})$ as well as $p_{\text {of }}$ and $p_{u f}$ all require the stationary distribution $\pi$, which may only be obtained by numerically solving the system of linear equations (4), given a specific state transition probability matrix $\mathbf{Q}$. Therefore, the optimisation problems (P1) and (P2) cannot be solved in polynomial time, when we have a high-level unary encoder.

We first provide an exhaustive search (ES) based algorithm for solving (P1), whose pseudo code is provided in Algorithm 1. Its complexity $O\left(\lfloor 1 / \varepsilon\rfloor^{K}\right)$ depends on the searching increment $\varepsilon$ and the level $K$. If we increase $\varepsilon$ for obtaining a more 


$$
\Phi(b, k)= \begin{cases}\sum_{\lambda=b}^{k}\left(\begin{array}{c}
k \\
\lambda-b
\end{array}\right)\left(\begin{array}{l}
k \\
\lambda
\end{array}\right) v^{\lambda-b}(1-v)^{k-\lambda+b} \frac{\left(1-\omega_{E, 10}\right)^{\lambda-1} \omega_{E, 10}^{k-1-\lambda}}{k}\left[(k-\lambda)\left(1-\omega_{E, 10}\right)\left(1-\omega_{E, 01}\right)+\lambda \omega_{E, 10} \omega_{E, 01}\right], & 0 \leq b \leq k, \\
\sum_{\lambda=0}^{k+b}\left(\begin{array}{c}
k \\
\lambda-b
\end{array}\right)\left(\begin{array}{l}
k \\
\lambda
\end{array}\right) v^{\lambda-b}(1-v)^{k-\lambda+b} \frac{\left(1-\omega_{E, 10}\right)^{\lambda-1} \omega_{E, 10}^{k-1-\lambda}}{k}\left[(k-\lambda)\left(1-\omega_{E, 10}\right)\left(1-\omega_{E, 01}\right)+\lambda \omega_{E, 10} \omega_{E, 01}\right], & -k \leq b \leq 0, \\
0, & \text { otherwise, }\end{cases}
$$

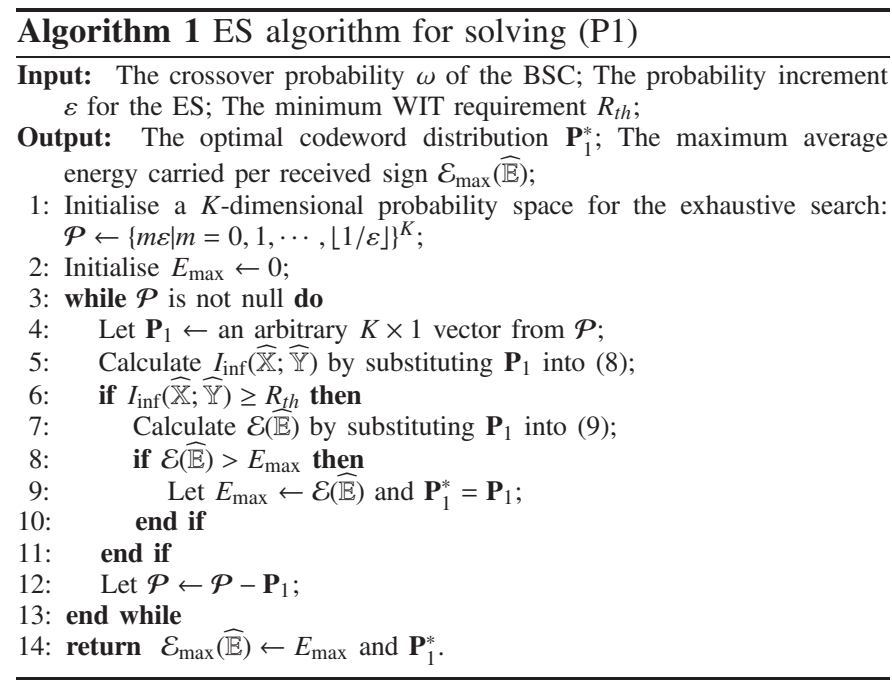

accurate result and $K$ for implementing high-level unary codes, the complexity increases dramatically. Algorithm 1 can also be slightly adjusted for solving (P2), which is omitted here for the page limitation.

\section{GA Aided Coding Design}

In order to reduce the computing complexity of the coding design but maintain its optimality, we then propose a GA aided coding design for solving the optimisation problem (P2). By exploiting both crossover and mutation treatments on the 'parents', GA aided coding design is capable of escaping from the local optimum, which is detailed in Algorithm 2. The main steps of Algorithm 2 can be summarised as below:

- Step 1: Randomly generate $N$ legitimate codeword distribution as a generation $\mathcal{P}$, as shown in Line 1 of Algorithm 2.

- Step 2: Calculate the corresponding mutual information and initialise overflow probabilities and the survival probabilities for all the codeword distributions in $\mathcal{P}$, as detailed in Lines 4-15 of Algorithm 2, while update the minimum overflow probability $p_{o f \text {,min }}$ expressed in the objective (21) of (P2), as shown in Lines 16-18 of Algorithm 2 . If the mutual information of an individual codeword distribution violates the minimum rate requirement $R_{t h}$ of the (P2)'s constraint (21a), its corresponding overflow probabilities are set to 1 . According to Line 14, this individual has a zero survival probability.

- Step 3: Randomly select individuals from $\mathcal{P}$ according to their survival probabilities $\mathbf{p}_{\text {live }}$ in order to generate the parental individuals $\mathcal{P}_{\text {sel }}$ for the sake of giving birth to the next generation, as detailed in Lines 19-26 of Algorithm $\overline{\text { Algorithm } 2 \text { GA aided optimal coding design for solving the }}$ optimisation problem (P2)

Input: The crossover probability $\omega$ of the BSC; The minimum WIT requirement $R_{t h}$; The maximum number of generations $G$; The population of a single generation $N$;

Output: The minimum overflow probabilities $p_{o f, \min }$; The optimal codeword distribution $\mathbf{P}_{2}^{*}$;

1: Randomly initialise a generation $\mathcal{P} \leftarrow\left\{\mathbf{P}_{(j)} \mid j=1, \cdots, N\right\}$, where $\mathbf{P}_{(j)}$ is a legitimate codeword distribution;

2: Initialise a generation index $g \leftarrow 1$ and the minimum overflow probability $p_{o f, \min } \leftarrow 1$;

3: while $g \leq G$ do

4: $\quad$ Initialise the mutual information of the $g$-th generation $\mathcal{I} \leftarrow\left\{I_{\text {inf, }(j)} \mid j=\right.$ $1, \cdots, N\}$, where $I_{\text {inf, }(j)}$ is calculated by substituting $\mathbf{P}_{(j)}$ into Eq. (8);

5: Initialise the overflow probabilities of the $g$-th generation $\mathbf{p}_{\text {of }} \leftarrow$ $\left\{p_{o f,(j)} \leftarrow 1 \mid j=1, \cdots, N\right\}$;

6: Initialise the survival probabilities of the $g$-th generation $\mathbf{p}_{\text {live }} \leftarrow$ $\left\{p_{\text {live },(j)} \leftarrow 0 \mid j=1, \cdots, N\right\}$;

7: $\quad$ Initialise the selected parental individuals $\mathcal{P}_{\text {sel }} \leftarrow\left\{\mathbf{P}_{\text {sel, }(j)} \leftarrow \mathbf{P}_{(j)} \mid j=\right.$ $1, \cdots, N\}$

8: for $\forall j=1, \cdots, N$ do

9: $\quad$ if $I_{\text {inf, }(j)} \geq R_{t h}$ then

10: $\quad$ Update $p_{o f,(j)}$ by substituting $\mathbf{P}_{(j)}$ into Eq. (19);

11: else

12: $\quad$ Update $p_{o f,(j)} \leftarrow 1$;

13: $\quad$ end if

14: $\quad$ Update $p_{\text {live },(j)} \leftarrow \frac{\left(1-p_{o f,(j)}\right)}{\sum_{j}\left(1-p_{o f,(j)}\right)}$;

15: end for

if $\min \mathbf{p}_{o f}<p_{o f, \min }$ then

Update $p_{o f, \min } \leftarrow \min \mathbf{p}_{\text {of }}$ and $\mathbf{P}_{2}^{*} \leftarrow \arg \min _{\mathbf{P}_{j}} \mathbf{p}_{o f}$

end if

for $\forall j=1, \cdots, N$ do

Generate $\zeta$ uniformly distributed in $[0,1]$;

Initialise a temporary index $n \leftarrow 1$;

while $\zeta-p_{\text {live, }(n)} \geq 0$ do

Update $\zeta \leftarrow \zeta-p_{\text {live }(n)}$ and $n \leftarrow n+1$;

end while

Update $\mathbf{P}_{s e l,(j)} \leftarrow \mathbf{P}_{(n)}$;

end for

Obtain a new generation $\mathcal{P}^{\prime}$ by inputting $\mathcal{P}_{\text {sel }}$ into Algorithm 3 and update $\mathcal{P} \leftarrow \mathcal{P}^{\prime}$;

28: end while

29: return $p_{o f, \min }$ and $\mathbf{P}_{2}^{*}$.

2. The individuals having lower overflow probabilities can be selected with higher probabilities, which is in line with the objective (21) of (P2).

- Step 4: Obtain a new generation $\mathcal{P}^{\prime}$ by carrying out crossover and mutation on the parental individuals $\mathcal{P}_{\text {sel }}$ in Algorithm 3 and repeat from Step 2, until we obtain the minimum overflow probability $p_{o f, \min }$ and the optimal codeword distribution $\mathbf{P}_{2}^{*}$ for (P2).

Furthermore, Algorithm 3 provides the details of crossover and mutation operations on the parental individuals. All the $N$ child individuals are born by the crossover of randomly chosen parents from $\mathcal{P}_{\text {sel }}$, as shown in Lines 2-4 of Algorithm 3. Every child individual has a probability of $\varepsilon$ to mutate, as illustrated in Lines 5-13 of Algorithm 3. Specifically, Lines 8- 


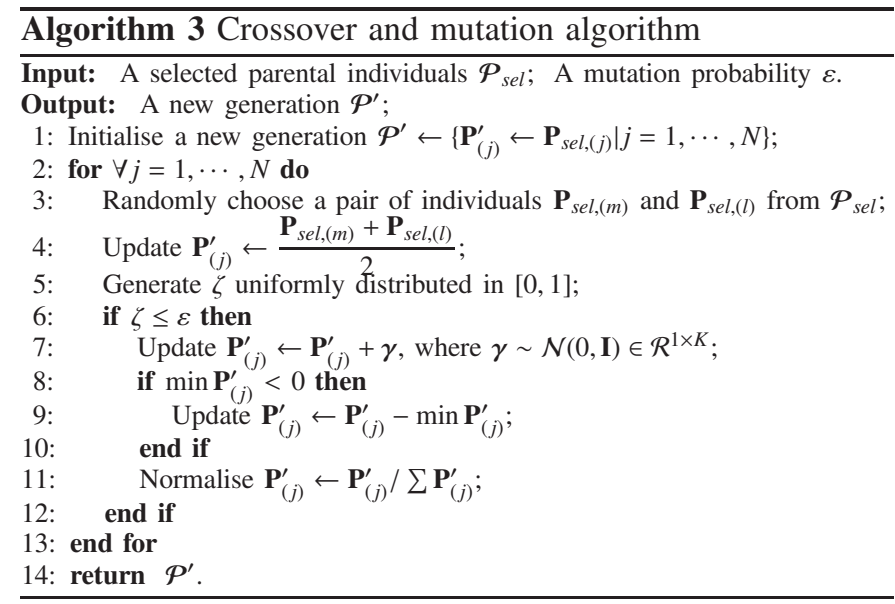

11 ensure that the mutated individual is a legitimate codeword distribution.

The complexity of the proposed GA aided optimal coding design is $O\left(G^{2}\right)$, where $G$ is the number of generations in Algorithm 2. The complexity is irrelevant to the level $K$ of the unary code. Therefore, when a high-level unary code is adopted for the SWIPT, the GA aided optimal coding design is capable of substantially reducing the computational complexity. Furthermore, by substituting $p_{\text {of }}$ in Algorithm 2 by $p_{u f}$, we can readily obtain the optimal codeword distribution for minimising the battery underflow probability. Moreover, Algorithm 2 can also be slightly adjusted for solving (P1), which is omitted here for the page limitation.

\section{Numerical Results}

In our simulation, we consider co-located IR and ER as a SWIPT receiver, whose distance from the SWIPT transmitter is $d_{E}=d_{I}=d=9.4 \mathrm{~m}$. The path-loss from the SWIPT transmitter to the receiver is modelled as $\Omega(d)=G_{t} G_{r}\left(\frac{4 \pi d f_{c}}{c}\right)^{2}$, where the antenna gains are set to $G_{t}=G_{r}=1$, the carrier frequency is $f_{c}=800 \mathrm{MHz}$ and $c$ is the speed of the light. The transmit power of the SWIPT transmitter is set to $P_{t}=20$ $\mathrm{dBm}$, while the sensitivity of the energy harvester is $P_{t h}=-30$ $\mathrm{dBm}$. Generally, the power of the ambient RF interference on both IR and ER are $\sigma_{I}^{2}=\sigma_{E}^{2}=\sigma^{2}$ varying from $-40 \mathrm{dBm}$ to $-25 \mathrm{dBm}$, since IR and ER are co-located.

\section{A. GA vs ES}

First of all, we demonstrate the convergence of the GA aided coding design and compare its performance to that of the ES aided counterpart in Fig.5. All the performance of Fig. 5 is attained by averaging over 50 random results. Observe from Figs. 5(a) and (b) that the GA aided coding design converges to the optimality in terms of both $\mathcal{E}_{\max }(\mathbb{E})$ and $p_{\min , u f}$, as the generation number increases. Furthermore, as shown in Fig. 5(a), after 10 generations, the GA aided coding design with medium population $N=100$ achieves the WPT performance of $\mathcal{E}_{\max }(\mathbb{\mathbb { E }})=0.3461$ energy unit/sign, only $3.1 \%$ lower than that of the ES aided counterpart. Moreover, as shown in Fig. 5 (b), the minimum underflow probability $p_{\min , u f}=0.0526$ is attained by the GA with $N=100$ after 10 generations, which

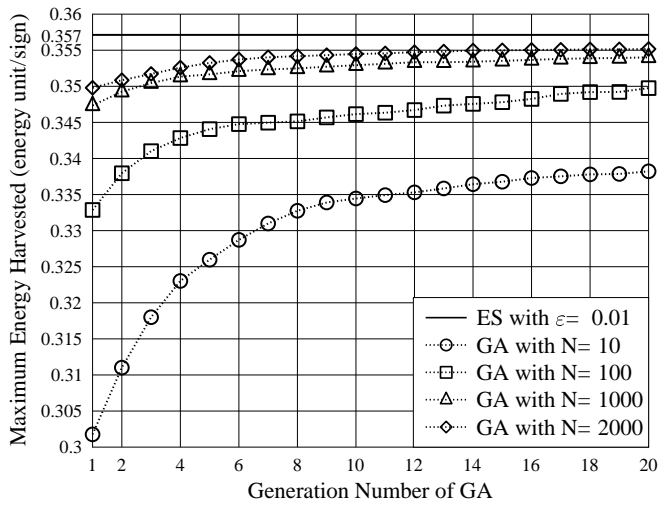

(a)

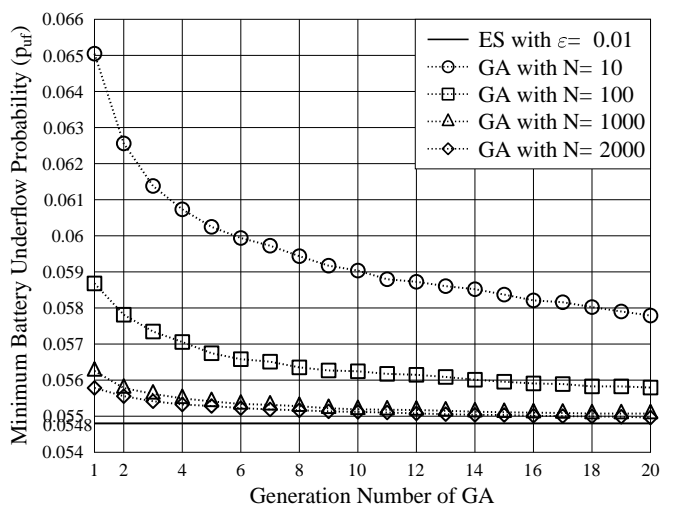

(b)

Fig. 5. Convergence of the GA aided coding design with different population $N=\{10,100,1000,2000\}$, where the 4-level unary code is adopted, the ambient RF interference power is $\sigma^{2}=-40 \mathrm{dBm}$ and the minimum WIT requirement is $R_{t h}=0.2$ bit/sign: (a) The maximum WPT performance $\mathcal{E}_{\max }(\widehat{\mathbb{E}})$ with infinite battery capacity and (b) the minimum underflow probability $p_{\min , u f}$ with finite battery capacity, which is in line with the setting in Section VI-C.

is $2.6 \%$ higher than that of the ES aided counterpart. Since the low-complexity GA aided coding design achieves almost the same performance with the ES, the rest of the numerical results are obtained by invoking GA.

\section{B. Infinite Battery Capacity}

We then investigate the SWIPT performance by considering an infinite battery capacity in Fig. 6, where the WPT performance is characterised by the average energy units harvested from a single received sign. Observe from both Figs. 6(a) and (b) that the maximum WPT performance $\mathcal{E}_{\max }(\widehat{\mathbb{E}})$ reduces, as we increase the WIT requirement $R_{t h}$. Note that $R_{t h}$ cannot exceed the channel capacity due to the limited WIT capability of a specific codeword distribution. For instance, as illustrated in Fig. 6(a), the maximum WIT capability of 2-level unary code is $0.47 \mathrm{bit} / \mathrm{sign}$. When we have $R_{\mathrm{th}}=0.47 \mathrm{bit} / \mathrm{sign}$, the WPT performance reaches its lowest, only 0.111 energy unit/sign. We may further observe from Fig. 6(a) that the 8level unary code has supreme SWIPT performance. Moreover, observe from Fig. 6(b) that the ambient RF interference power is helpful for WPT but it is harmful for WIT. For instance, when we have $\sigma^{2}=-25 \mathrm{dBm}$, the highest WPT performance is achieved, but the WIT performance of the 4-level unary 


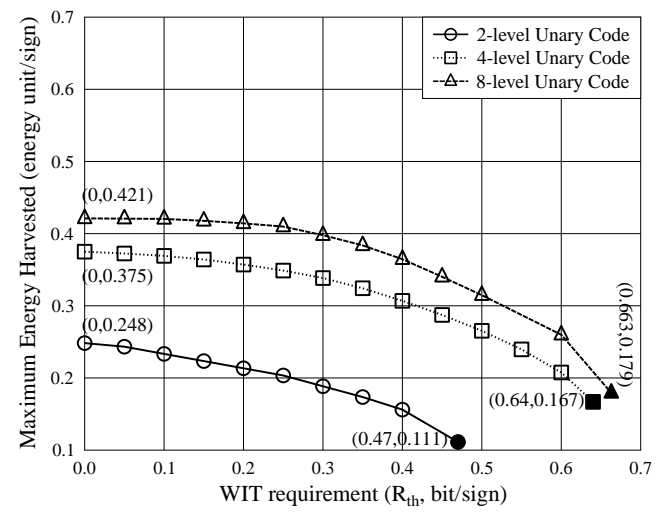

(a)

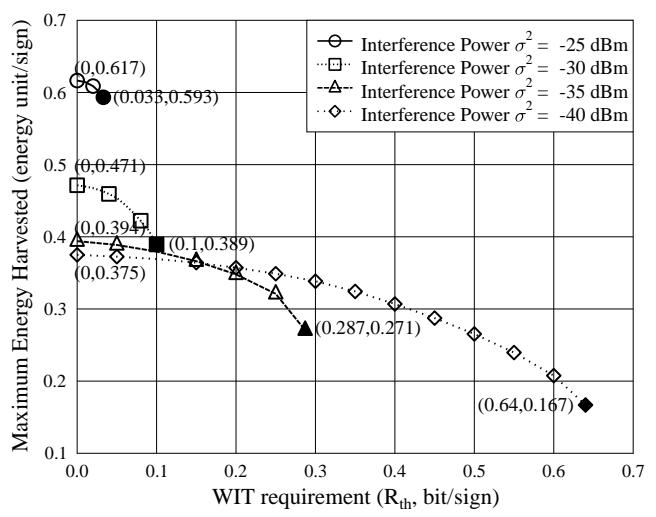

(b)

Fig. 6. The SWIPT performance with an infinite battery capacity: (a) $(2,4,8)$-level unary codes with the ambient RF interference power $\sigma^{2}=-40$ $\mathrm{dBm}$; (b) 4-level unary code with the ambient RF interference power $\sigma^{2}=$ $\{-25,-30,-35,-40\} \mathrm{dBm}$. The filled markers represent the conventional WIT coding schemes, which aims for maximising the mutual information of Eq. (8) by ignoring the QoS requirement of WPT.

code is very limited, which is lower than 0.033 bit/sign. Furthermore, when $R_{t h}$ is lower than $0.15 \mathrm{bit} / \mathrm{sign}$, the WPT performance associated with $\sigma^{2}=-35 \mathrm{dBm}$ is higher than that with $\sigma^{2}=-40 \mathrm{dBm}$. By contrast, if the $R_{t h}$ is between 0.15 and $0.64 \mathrm{bit} / \mathrm{sign}$, the channel having $\sigma^{2}=-40 \mathrm{dBm}$ is capable of transferring more energy to the SWIPT receiver.

\section{Finite Battery Capacity}

We then consider finite battery capacity having $B_{\max }=2$ energy units and $B_{\min }=0$ energy units, while the ER consumes a single energy unity with a probability of $v=0.5$ within a sign duration. Observe from Figs. 7(a) and (b) that both the battery underflow and overflow probabilities increases, as the WIT requirement becomes stringent. Since the unary codeword distribution has to be adjusted in order to satisfy the harsh WIT requirement, the battery underflow and overflow probabilities are inevitably sacrificed during the coding design. Furthermore, as illustrated in Fig. 7(a), a higher level of unary code has higher freedom for the sake of satisfying harsher WIT requirements and reaching better WPT performance. Moreover, observe from Fig. 7(b) that when the ambient RF interference power $\sigma^{2}$ becomes higher, the WIT performance becomes worse, since it impairs the information demodulation

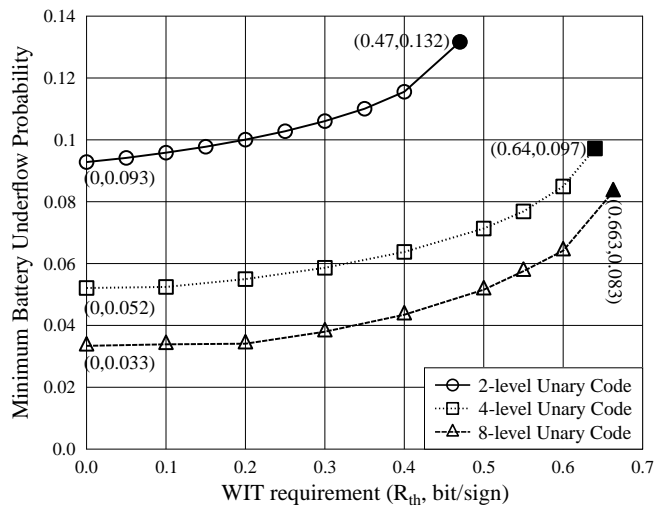

(a)

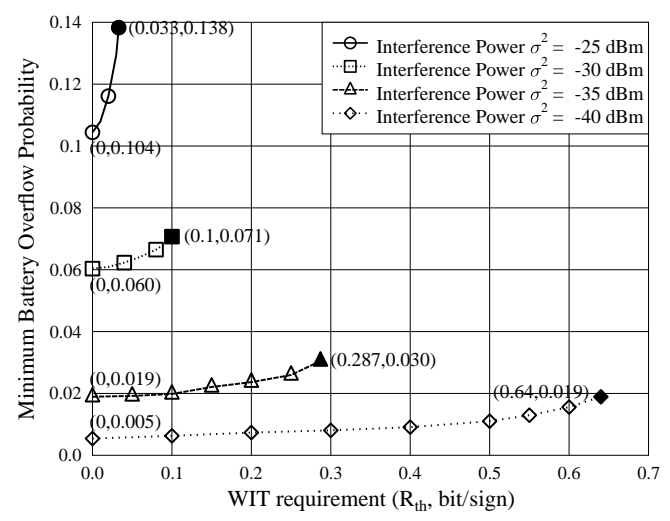

(b)

Fig. 7. The SWIPT performance with a finite battery capacity: (a) $(2,4,8)$ level unary codes with the ambient RF interference power $\sigma^{2}=-40$ $\mathrm{dBm}$; (b) 4-level unary code with the ambient RF interference power $\sigma^{2}=$ $\{-25,-30,-35,-40\} \mathrm{dBm}$. The filled markers represent the conventional WIT coding schemes, which aims for maximising the mutual information of Eq. (8) by ignoring the QoS requirement of WPT.

of the IR, while the WPT performance becomes better, since ambient RF interference provides more energy to the ER.

\section{Conclusion}

A unary coded SWIPT transceiver is studied in this paper by considering practical WIT and WPT channels. The unary coded transmitter is modelled by a Markov chain having finite states, which is relied upon for the WIT and WPT performance analysis. The optimal codeword distribution is found by exploiting the low-complexity GA for maximising the average energy harvested from a single received sign and for minimising the battery overflow/underflow probability, respectively, by satisfying the minimum requirement of the information transmission rate. The numerical results demonstrate the feasibility of the GA aided coding design and they explicitly characterise the tradeoff between the WIT and the WPT in the coding level.

Coding controlled SWIPT can be readily implemented in the upper layer, when physical transmission mechanism of a practical communication system cannot be customised. In the future, we will focus our attention on the following two aspects of the coding controlled SWIPT: 1) an optimal encoding scheme for mapping original messages onto the 
codewords by considering data compression, distortion and SWIPT performance; 2) joint design of coding and modulation for SWIPT, when M-QAM is conceived.

\section{Appendix A \\ Proof of Lemma 1}

The Markov chain of Fig. 2 transits from the state $S_{i}$ to $S_{i+1}$ due to the coding principle of the unary encoder. By contrast, the transition from the state $S_{i}$ to the $\operatorname{sign} Y_{i}$ is induced by the imperfect information transmission in the WIT channel of Fig. 1. Obviously, the transition process from the state $S_{i}$ to the state $S_{i+1}$ is physically uncorrelated to the transition process from the state $S_{i}$ to $Y_{i}$, which can be expressed as the following equality ${ }^{2}$ :

$$
p\left(y_{i}, s_{i+1} \mid s_{i}\right)=p\left(y_{i} \mid s_{i}\right) \cdot p\left(s_{i+1} \mid s_{i}\right) .
$$

Moreover, the conditional probability $p\left(y_{i}, s_{i+1} \mid s_{i}\right)$ can be further expressed as

$$
p\left(y_{i}, s_{i+1} \mid s_{i}\right)=p\left(y_{i} \mid s_{i+1}, s_{i}\right) \cdot p\left(s_{i+1} \mid s_{i}\right)=p\left(y_{i} \mid s_{i}\right) \cdot p\left(s_{i+1} \mid s_{i}\right),
$$

where the second equality is derived by substituting Eq. (22) into Eq. (23). Therefore we have $p\left(y_{i} \mid s_{i+1}, s_{i}\right)=p\left(y_{i} \mid s_{i}\right)$. By multiplying $p\left(s_{i}\right)$ on both sides of this equation and summing them over all possible values of $s_{i}$, we may derive the following equalities ${ }^{3}$

$$
\begin{aligned}
& \sum_{s_{i}} p\left(y_{i} \mid s_{i+1}, s_{i}\right) \cdot p\left(s_{i}\right)=\sum_{s_{i}} p\left(y_{i} \mid s_{i}\right) \cdot p\left(s_{i}\right) \\
\Rightarrow & \sum_{s_{i}} p\left(y_{i}, s_{i} \mid s_{i+1}\right)=\sum_{s_{i}} p\left(y_{i}, s_{i}\right) \Rightarrow p\left(y_{i} \mid s_{i+1}\right)=p\left(y_{i}\right) \\
\Rightarrow & p\left(y_{i} \mid s_{i+1}\right) \cdot p\left(s_{i+1}\right)=p\left(y_{i}\right) \cdot p\left(s_{i+1}\right) \\
\Rightarrow & p\left(y_{i}, s_{i+1}\right)=p\left(y_{i}\right) \cdot p\left(s_{i+1}\right),
\end{aligned}
$$

which demonstrates that the random event $Y_{i}=y_{i}$ is independent of the random event $S_{i+1}=s_{i+1}$.

We may further prove that the channel output signs $\left\{Y_{1}, \cdots, Y_{i-1}\right\}$ are all uncorrelated to the state $S_{i+1}$. Firstly, $\left\{Y_{1}, \cdots, Y_{i-1}\right\}$ only depends on the corresponding states $\left\{S_{1}, \cdots, S_{i-1}\right\}$ at the same instants. Secondly, when $S_{i}$ is given, the state $S_{i+1}$ only depends on $S_{i}$ but it is independent of all the previous states due to the Markov property. Therefore, Lemma 1 is proved.

\section{APPENDIX B}

Proof of Lemma 2

The basic principle of proving this lemma is to find the inequalities of $\left\{p\left(S_{i}=s_{i} \mid Y_{i+n}=y_{i+n}\right) \neq p\left(S_{i}=s_{i}\right) \mid n=\right.$ $1,2, \cdots$,$\} , where s_{i} \in\left\{0,1, \cdots, 2^{k}-1\right\}$ and $y_{i+n} \in\{0,1\}$.

1) We first prove that the random event $S_{i}=s_{i}$ depends on the random event $Y_{i+1}=y_{i+1}$.

The joint probability of these two random events is $p\left(s_{i}, y_{i+1}\right)=p\left(s_{i}\right) \cdot p\left(y_{i+1} \mid s_{i}\right)$. Furthermore, the probability of

\footnotetext{
${ }^{2}$ The capital letter $S_{i}$ represents a random variable and $s_{i}$ represents a possible value of this random variable.

${ }^{3} p\left(s_{i}\right)$ is the probability of the Markov state machine staying at the state $s_{i}$. When the Markov chain becomes stationary, we have $p\left(s_{i}\right)=\pi_{s_{i}}$.
}

the random event $Y_{i+1}=y_{i+1}$ occurring is $p\left(y_{i+1}\right)=\sum_{s_{i}} p\left(s_{i}\right)$. $p\left(y_{i+1} \mid s_{i}\right)$. Therefore, the conditional probability $p\left(s_{i} \mid y_{i+1}\right)$ can be obtained as

$$
p\left(s_{i} \mid y_{i+1}\right)=\frac{p\left(s_{i}, y_{i+1}\right)}{p\left(y_{i+1}\right)}=\frac{p\left(s_{i}\right) \cdot p\left(y_{i+1} \mid s_{i}\right)}{\sum_{s_{i}} p\left(s_{i}\right) \cdot p\left(y_{i+1} \mid s_{i}\right)} .
$$

When $y_{i+1}=1$, the conditional probability $p\left(y_{i+1}=1 \mid s_{i}\right)$ of the WIT channel is expressed as

$$
p\left(y_{i+1}=1 \mid s_{i}\right)=q_{s_{i}} \cdot\left(1-\omega_{I}\right)+\left(1-q_{s_{i}}\right) \cdot \omega_{I},
$$

where $q_{s_{i}}$ is the transition probability from the state $s_{i}$ to $\left(s_{i}+1\right)$, which is also the probability of the unary encoder inputting a sign ' 1 ' to the WIT channel. This sign ' 1 ' then has a probability of $\left(1-\omega_{I}\right)$ to be unchanged by the WIT channel. Moreover, the probability of a sign ' 0 ' being input to the WIT channel is $\left(1-q_{s_{i}}\right)$, while this sign is changed to sign ' 1 ' with a probability of $\omega_{I}$. Substituting Eq. (26) into (25), we have

$$
p\left(s_{i} \mid y_{i+1}=1\right)=\frac{p\left(s_{i}\right) \cdot\left(q_{s_{i}}-2 q_{s_{i}} \omega_{I}+\omega_{I}\right)}{\sum_{s_{i}} p\left(s_{i}\right) \cdot\left(q_{s_{i}}-2 q_{s_{i}} \omega_{I}+\omega_{I}\right)} .
$$

Observe from Eq. (27) that $\left\{q_{s_{i}}=q \mid s_{i}=0,1, \cdots, K-1\right\}$ is a feasible solution to let $p\left(s_{i} \mid y_{i+1}=1\right)=p\left(s_{i}\right)$, where $q \in[0,1]$ is an arbitrary constant. Otherwise, we have $p\left(s_{i} \mid y_{i+1}=1\right) \neq$ $p\left(s_{i}\right)$.

When $y_{i+1}=0$, the conditional probability $p\left(y_{i=1}=0 \mid s_{i}\right)$ of the WIT channel is expressed as

$$
p\left(y_{i=1}=0 \mid s_{i}\right)=q_{s_{i}} \omega_{I}+\left(1-q_{s_{i}}\right)\left(1-\omega_{I}\right) .
$$

This is because the unary encoder inputs a sign ' 1 ' to the WIT channel, when the Markov chain of Fig. 2 transits from $s_{i}$ to $s_{i+1}$ with a probability of $q_{s_{i}}$. This sign ' 1 ' has a probability of $\omega_{I}$ to be changed to ' 0 ' by the WIT channel. Moreover, the probability of a sign ' 0 ' being input is $\left(1-q_{s_{i}}\right)$, while this sign is unchanged by the WIT channel with a probability of $\left(1-\omega_{I}\right)$. Substituting Eq. (28) into (25), we have

$$
p\left(s_{i} \mid y_{i+1}=0\right)=\frac{p\left(s_{i}\right) \cdot\left[q_{s_{i}} \omega_{I}+\left(1-q_{s_{i}}\right)\left(1-\omega_{I}\right)\right]}{\sum_{s_{i}} p\left(s_{i}\right) \cdot\left[q_{s_{i}} \omega_{I}+\left(1-q_{s_{i}}\right)\left(1-\omega_{I}\right)\right]} .
$$

Observe from both Eqs. (27) and (29) that $\left\{q_{s_{i}}=q \mid s_{i}=\right.$ $0,1, \cdots, K-1\}$ is a feasible solution to simultaneously let $p\left(s_{i} \mid y_{i+1}=1\right)=p\left(s_{i}\right)$ and $p\left(s_{i} \mid y_{i+1}=0\right)=p\left(s_{i}\right)$, which indicates that the random event $S_{i}=s_{i}$ is independent of $Y_{i+1}=y_{i+1}$. Otherwise, $S_{i}=s_{i}$ and $Y_{i+1}=y_{i+1}$ are correlated with each other, since we have $p\left(s_{i} \mid y_{i+1}\right) \neq p\left(s_{i}\right)$.

2) We then prove that the random event $S_{i}=s_{i}$ depends on the random event $Y_{i+2}=y_{i+2}$ :

The joint probability of these two random events is $p\left(s_{i}, y_{i+2}\right)=\sum_{s_{i+1}} \sum_{s_{i+2}} p\left(s_{i}, s_{i+1}, s_{i+2}\right) \cdot p\left(y_{i+2} \mid s_{i}, s_{i+1}, s_{i+2}\right)$. Furthermore, the probability of the random event $Y_{i+2}=y_{i+2}$ occurring is $p\left(y_{i+2}\right)=\sum_{s_{i}} p\left(s_{i}, y_{i+2}\right)$. Therefore, the conditional probability $p\left(s_{i} \mid y_{i+2}\right)$ can be obtained as

$$
p\left(s_{i} \mid y_{i+2}\right)=\frac{p\left(s_{i}, y_{i+2}\right)}{p\left(y_{i+2}\right)}=\frac{p\left(s_{i}, y_{i+2}\right)}{\sum_{s_{i}} p\left(s_{i}, y_{i+2}\right)} .
$$

When the output sign of the WIT channel at the $(i+2)$-th instant is $y_{i+2}=1$, the joint probability $p\left(s_{i}, y_{i+2}=1\right)$ can be 
formulated as

$$
\begin{aligned}
p\left(s_{i}, y_{i+2}=1\right)= & p\left(s_{i}, s_{i}+1, s_{i}+2\right) p\left(y_{i+2}=1 \mid s_{i}, s_{i}+1, s_{i}+2\right) \\
& +p\left(s_{i}, s_{i}+1,0\right) p\left(y_{i+2}=1 \mid s_{i}, s_{i}+1,0\right) \\
& +p\left(s_{i}, 0,1\right) p\left(y_{i+2}=1 \mid s_{i}, 0,1\right) \\
& +p\left(s_{i}, 0,0\right) p\left(y_{i+2}=1 \mid s_{i}, 0,0\right) \\
= & p\left(s_{i}\right)\left\{\left(1-\omega_{I}\right)\left[q_{s_{i}} q_{s_{i}+1}+\left(1-q_{s_{i}}\right) q_{0}\right]\right. \\
& \left.+\omega_{I}\left[q_{s_{i}}\left(1-q_{s_{i}+1}\right)+\left(1-q_{s_{i}}\right)\left(1-q_{0}\right)\right]\right\} . \quad(31)
\end{aligned}
$$

This is because when $s_{i+2} \neq 0$, the unary encoder inputs a sign ' 1 ' to the WIT channel, which is unchanged with a probability of $\left(1-\omega_{I}\right)$. When $s_{i+2}=0$, a sign ' 0 ' is input to the WIT channel, which is changed with a probability of $\omega_{I}$. Substituting Eq. (31) into (30), we have

$$
p\left(s_{i} \mid y_{i+2}=1\right)=\frac{p\left(s_{i}, y_{i+2}=1\right)}{\sum_{s_{i}} p\left(s_{i}, y_{i+2}=1\right)} .
$$

Observe from Eqs. (31) and (32) that $\left\{q_{s_{i}}=q \mid s_{i}=0,1, \cdots, K-\right.$ $1\}$ is able to let $p\left(s_{i} \mid y_{i+2}=1\right)=p\left(s_{i}\right)$, where $q \in[0,1]$ is an arbitrary constant. Otherwise, we have $p\left(s_{i} \mid y_{i+2}=1\right) \neq p\left(s_{i}\right)$.

When the output sign of the WIT channel at the $(i+2)$-th instant is $y_{i+2}=0$, the joint probability $p\left(s_{i}, y_{i+2}=0\right)$ can be formulated as

$$
\begin{aligned}
p\left(s_{i}, y_{i+2}=0\right)= & p\left(s_{i}, s_{i}+1, s_{i}+2\right) p\left(y_{i+2}=0 \mid s_{i}, s_{i}+1, s_{i}+2\right) \\
& +p\left(s_{i}, s_{i}+1,0\right) p\left(y_{i+2}=0 \mid s_{i}, s_{i}+1,0\right) \\
& +p\left(s_{i}, 0,1\right) p\left(y_{i+2}=0 \mid s_{i}, 0,1\right) \\
& +p\left(s_{i}, 0,0\right) p\left(y_{i+2}=0 \mid s_{i}, 0,0\right) \\
= & p\left(s_{i}\right)\left[q_{s_{i}} q_{s_{i}+1} \omega_{I}+q_{s_{i}}\left(1-q_{s_{i}+1}\right)\left(1-\omega_{I}\right)\right. \\
& \left.+\left(1-q_{s_{i}}\right) q_{0} \omega_{I}+\left(1-q_{s_{i}}\right)\left(1-q_{0}\right)\left(1-\omega_{I}\right)\right],
\end{aligned}
$$

This is because when $s_{i+2} \neq 0$, the unary encoder inputs a sign ' 1 ' to the WIT channel, which is changed to ' 0 ' with a probability of $\omega_{I}$. When $s_{i+2}=0$, a sign ' 0 ' is input to the WIT channel, which is unchanged with a probability of $\omega_{I}$. Substituting Eq. (33) into (30), we have

$$
p\left(s_{i} \mid y_{i+2}=0\right)=\frac{p\left(s_{i}\right)\left[1-\left(1-\omega_{I}\right)\left(q_{s_{i}} q_{s_{i}+1}+q_{0}-q_{s_{i}} q_{0}\right)\right]}{\sum_{s_{i}} p\left(s_{i}\right)\left[1-\left(1-\omega_{I}\right)\left(q_{s_{i}} q_{s_{i}+1}+q_{0}-q_{s_{i}} q_{0}\right)\right]},
$$

Observe from Eqs. (31) and (32) that $\left\{q_{s_{i}}=q \mid s_{i}=0,1, \cdots, K-\right.$ $1\}$ is also a feasible solution to let $p\left(s_{i} \mid y_{i+2}=0\right)=p\left(s_{i}\right)$, where $q \in[0,1]$ is an arbitrary constant. Otherwise, we have $p\left(s_{i} \mid y_{i+2}=0\right) \neq p\left(s_{i}\right)$. Therefore, the random event $S_{i}=s_{i}$ and $Y_{i+2}=y_{i+2}$ are independent, only if we have $\left\{q_{s_{i}}=q \mid s_{i}=\right.$ $0,1, \cdots, K\}$. Otherwise, they are correlated with each other.

Relying on the similar methodology, we may also prove that $S_{i}=s_{i}$ is correlated with $Y_{i+n}=y_{i+n}$ for $\forall n \geq 1$. Furthermore, $\left\{q_{s_{i}}=q \mid s_{i}=0,1, \cdots, K-1\right\}$ is a feasible solution to let $S_{i}=s_{i}$ simultaneously independent of $\left\{Y_{i+1}=y_{i+1}, Y_{i+2}=y_{i+2}, \cdots\right\}$. Lemma 2 is proved.

\section{REFERENCES}

[1] Z. Dawy, W. Saad, A. Ghosh, J. G. Andrews, and E. Yaacoub, "Toward Massive Machine Type Cellular Communications," IEEE Wireless Communications, vol. 24, no. 1, pp. 120-128, February 2017.
[2] J. Hu, K. Yang, G. Wen, and L. Hanzo, "Integrated Data and Energy Communication Network: A Comprehensive Survey," IEEE Communications Surveys Tutorials, early access, August 2018.

[3] I. Krikidis, S. Timotheou, S. Nikolaou, G. Zheng, D. W. K. Ng, and R. Schober, "Simultaneous wireless information and power transfer in modern communication systems," IEEE Communications Magazine, vol. 52, no. 11, pp. 104-110, Nov 2014.

[4] X. Zhou, R. Zhang, and C. K. Ho, "Wireless information and power transfer: Architecture design and rate-energy tradeoff," IEEE Transactions on Communications, vol. 61, no. 11, pp. 4754-4767, November 2013.

[5] B. Clerckx and E. Bayguzina, "Waveform Design for Wireless Power Transfer," IEEE Transactions on Signal Processing, vol. 64, no. 23, pp. 6313-6328, Dec 2016.

[6] Y. Zeng, B. Clerckx, and R. Zhang, "Communications and Signals Design for Wireless Power Transmission," IEEE Transactions on Communications, vol. 65, no. 5, pp. 2264-2290, May 2017.

[7] X. Zhou, R. Zhang, and C. K. Ho, "Wireless Information and Power Transfer: Architecture Design and Rate-Energy Tradeoff," IEEE Transactions on Communications, vol. 61, no. 11, pp. 4754-4767, November 2013.

[8] R. Zhang and C. K. Ho, "MIMO Broadcasting for Simultaneous Wireless Information and Power Transfer," IEEE Transactions on Wireless Communications, vol. 12, no. 5, pp. 1989-2001, May 2013.

[9] K. Lv, J. Hu, Q. Yu, and K. Yang, "Throughput Maximization and Fairness Assurance in Data and Energy Integrated Communication Networks," IEEE IoT Journal, vol. 5, no. 2, pp. 636-644, April 2018.

[10] J. Yang, J. Hu, K. Lv, Q. Yu, and K. Yang, "Multi-Dimensional Resource Allocation for Uplink Throughput Maximisation in Integrated Data and Energy Communication Networks," IEEE Access, vol. 6, pp. $47163-$ $47180,2018$.

[11] S. Gautam, E. Lagunas, S. Chatzinotas, and B. Ottersten, "Relay selection and resource allocation for swipt in multi-user ofdma systems," IEEE Transactions on Wireless Communications, vol. 18, no. 5, pp. 2493-2508, May 2019.

[12] Y. Zhao, J. Hu, Y. Diao, Q. Yu, and K. Yang, "Modelling and Performance Analysis of Wireless LAN Enabled by RF Energy Transfer,' IEEE Transactions on Communications, vol. 66, no. 11, pp. 5756-5772, Nov 2018.

[13] Y. Zhao, J. Hu, R. Guo, K. Yang, and S. Leng, "Enhanced CSMA/CA Protocol Design for Integrated Data and Energy Transfer in WLANs," in proceedings of IEEE Globecom 2018, Dec 2018.

[14] Y. Zhao, D. Wang, J. Hu, and K. Yang, "H-AP Deployment for Joint Wireless Information and Energy Transfer in Smart Cities," IEEE Transactions on Vehicular Technology, vol. 67, no. 8, pp. 7485-7496, Aug 2018

[15] A. M. Fouladgar and O. Simeone, "On the transfer of information and energy in multi-user systems," IEEE Communications Letters, vol. 16, no. 11, pp. 1733-1736, November 2012.

[16] J. Hu, Y. Zhao, and K. Yang, "Modulation and Coding Design for Simultaneous Wireless Information and Power Transfer," IEEE Communication Magazine, vol. 57, no. 5, May 2019.

[17] L. R. Varshney, "Transporting Information and Energy Simultaneously," in 2008 IEEE ISIT, Jul. 2008, pp. 1612-1616.

[18] — "On Energy/Information Cross-Layer Architectures," in 2012 IEEE International Symposium on Information Theory Proceedings. IEEE, Jul 2012, pp. 1356-1360.

[19] P. Popovski, A. M. Fouladgar, and O. Simeone, "Interactive joint transfer of energy and information," IEEE Transactions on Communications, vol. 61, no. 5, pp. 2086-2097, May 2013.

[20] S. Yin and Z. Qu, "Rate-optimal coding design in joint transfer of energy and information," IEEE Communications Letters, vol. 19, no. 5, pp. 715718, May 2015.

[21] A. Tandon, M. Motani, and L. R. Varshney, "Subblock-Constrained Codes for Real-Time Simultaneous Energy and Information Transfer," IEEE Trans. on Info. Theory, vol. 62, no. 7, pp. 4212-4227, Jul 2016.

[22] A. I. Barbero, E. Rosnes, G. Yang, and O. Ytrehus, "Constrained codes for passive RFID communication," in 2011 IEEE ITA, Feb. 2011, pp. $1-9$.

[23] A. M. Fouladgar, O. Simeone, and E. Erkip, "Constrained Codes for Joint Energy and Information Transfer," IEEE Trans. on Comm., vol. 62 , no. 6, pp. 2121-2131, Jun. 2014.

[24] A. Tandon, M. Motani, and L. R. Varshney, "On Code Design for Simultaneous Energy and Information Transfer," in 2014 Information Theory and Applications Workshop (ITA). IEEE, feb 2014, pp. 1-6. 
[25] Z. Babar, M. A. M. Izhar, H. V. Nguyen, P. Botsinis, D. Alanis, D. Chandra, S. X. Ng, R. G. Maunder, and L. Hanzo, "Unary-Coded Dimming Control Improves ON-OFF Keying Visible Light Communication," IEEE Trans. on Comm., vol. 66, no. 1, pp. 255-264, Jan. 2018.

[26] W. Zhang, Z. Song, M. F. Brejza, T. Wang, R. G. Maunder, and L. Hanzo, "Learning-Aided Unary Error Correction Codes for NonStationary and Unknown Sources," IEEE Access, vol. 4, pp. 2408-2428, 2016.

[27] W. Zhang, M. F. Brejza, T. Wang, R. G. Maunder, and L. Hanzo, "Irregular Trellis for the Near-Capacity Unary Error Correction Coding of Symbol Values From an Infinite Set," IEEE Transactions on Communications, vol. 63, no. 12, pp. 5073-5088, Dec 2015.

[28] T. M. Cover and J. A. Thomas, Elements of Information Theory, 2nd ed. John Wiley \& Sons, Inc., 2006

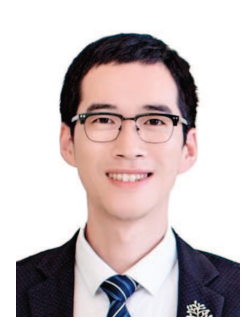

Jie Hu (S'11-M'16) received his B.Eng. and M.Sc. degrees from Beijing University of Posts and Telecommunications, China, in 2008 and 2011, respectively, and received the Ph.D. degree from the School of Electronics and Computer Science, University of Southampton, U.K., in 2015. Since March 2016, he has been working with the School of Information and Communication Engineering, University of Electronic Science and Technology of China (UESTC), China, as an Associate Professor. His research now is funded by National Natural Science Foundation of China (NSFC) and National Innovative Research Program. He is also in great partnership with industry, such as Huawei and ZTE. He has served for ZTE Communications as the guest editor of the special issue "Wireless Data and Energy Integrated Communication Networks". He also works as a TPC member in a number of prestigious IEEE conferences, such as IEEE ICC/Globecom/WSCP etc. He has a broad range of interests in wireless communication and networking, such as cognitive radio and cognitive networks, mobile social networks, simultaneous wireless information and power transfer as well as communication and computation convergence.

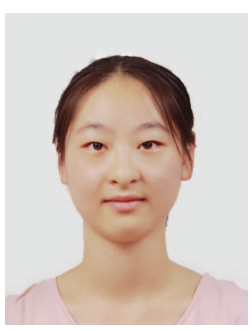

Mengyuan Li received her B.Eng. degree from Northwestern Polytechnical University in 2017. She is currently studying in the School of Information and Communication Engineering, UESTC for her master degree. Her research interests include information theory and coding applied to wireless information and energy transfer.

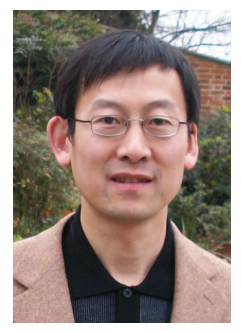

Kun Yang (SM'08) received his $\mathrm{PhD}$ from the Department of Electronic \& Electrical Engineering of University College London (UCL), UK, and MSc and BSc from the Computer Science Department of Jilin University, China. He is currently a Chair Professor in the School of Computer Science \& Electronic Engineering, University of Essex, leading the Network Convergence Laboratory (NCL), UK. He is also an affiliated professor at UESTC, China. Before joining in University of Essex at 2003, he worked at UCL on several European Union (EU) research projects for several years. His main research interests include wireless networks and communications, data and energy integrated networks, computation-communication cooperation. He manages research projects funded by various sources such as UK EPSRC, EU FP7/H2020 and industries. He has published $100+$ journal papers. He serves on the editorial boards of both IEEE and non-IEEE journals. He is a Senior Member of IEEE (since 2008) and a Fellow of IET (since 2009).

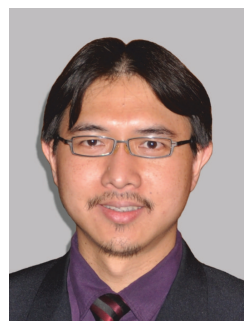

Soon Xin Ng ((S'99-M03-SM'08)) received the B.Eng. degree (First class) in electronic engineering and the Ph.D. degree in telecommunications from the University of Southampton, Southampton, U.K., in 1999 and 2002, respectively. From 2003 to 2006, he was a postdoctoral research fellow working on collaborative European research projects known as SCOUT, NEWCOM and PHOENIX. Since August 2006, he has been a member of academic staff in the School of Electronics and Computer Science, University of Southampton. He was involved in the OPTIMIX and CONCERTO European projects as well as the IU-ATC and UC4G projects. He was the principal investigator of an EPSRC project on "Cooperative Classical and Quantum Communications Systems". He is currently an Associate Professor in telecommunications at the University of Southampton.

His research interests include adaptive coded modulation, coded modulation, channel coding, space-time coding, joint source and channel coding, iterative detection, OFDM, MIMO, cooperative communications, distributed coding, quantum communications, quantum error correction codes, joint wireless-and-optical-fibre communications, game theory, artificial intelligence and machine learning. He has published over 250 papers and co-authored two John Wiley/IEEE Press books in this field.

$\mathrm{He}$ is a Senior Member of the IEEE, a Fellow of the Higher Education Academy in the UK, a Chartered Engineer and a Fellow of the IET. He acted as TPC/track/workshop chairs for various conferences. He serves as an associate editor to the IEEE Access and as the editor of Quantum Engineering. He was/is one of the guest editors for the special issues in: IEEE Journal on Selected Areas in Communications (2019) and IEEE Access (2017). He was also one of the editors in KSII Transactions on Internet and Information Systems. He is one of the Founders and Officers of the IEEE Quantum Communications \& Information Technology Emerging Technical Subcommittee (QCIT-ETC).

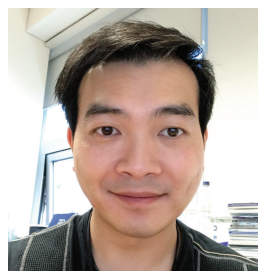

Kai-Kit Wong ((M'01-SM'08-F'16)) received the BEng, the MPhil, and the $\mathrm{PhD}$ degrees, all in Electrical and Electronic Engineering, from the Hong Kong University of Science and Technology, Hong Kong, in 1996, 1998, and 2001, respectively. After graduation, he took up academic and research positions at the University of Hong Kong, Lucent Technologies, Bell-Labs, Holmdel, the Smart Antennas Research Group of Stanford University, and the University of Hull, UK. He is Chair in Wireless Communications at the Department of Electronic and Electrical Engineering, University College London, UK.

His current research centers around $5 \mathrm{G}$ and beyond mobile communications, including topics such as massive MIMO, full-duplex communications, millimetre-wave communications, edge caching and fog networking, physical layer security, wireless power transfer and mobile computing, V2X communications, and of course cognitive radios. There are also a few other unconventional research topics that he has set his heart on, including for example, fluid antenna communications systems, remote ECG detection and etc. He is a co-recipient of the 2013 IEEE Signal Processing Letters Best Paper Award and the 2000 IEEE VTS Japan Chapter Award at the IEEE Vehicular Technology Conference in Japan in 2000, and a few other international best paper awards.

He is Fellow of IEEE and IET and is also on the editorial board of several international journals. He has served as Senior Editor for IEEE Communications Letters since 2012 and also for IEEE Wireless Communications Letters since 2016. He had also previously served as Associate Editor for IEEE Signal Processing Letters from 2009 to 2012 and Editor for IEEE Transactions on Wireless Communications from 2005 to 2011. He was also Guest Editor for IEEE JSAC SI on virtual MIMO in 2013 and currently Guest Editor for IEEE JSAC SI on physical layer security for $5 \mathrm{G}$. 